\title{
«Nicht unsere Körper sind das Problem, sondern die Barrieren!»
}

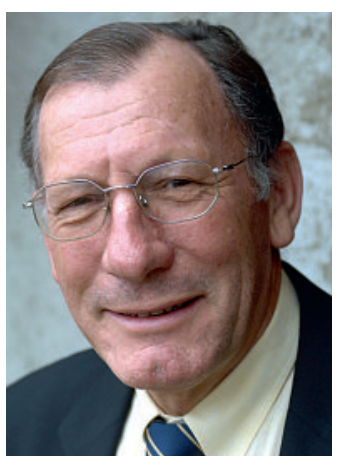

Jean Martin

1 Shakespeare T. Disability, genetics and global justice. Social Policy and Society. 2005; 4(1):87-95.

2 Martin J. Méthodes amélioratives (enhancement) et autres avancées. Revue médicale suisse. 2009; 5:606

3 Martin J. Die Unsterblichkeit - welch Albtraum. Schweiz Ärztezeitung. 2009;90(3):90.

4 Auch hier eine Anmerkung aus dem Bereich der öffentlichen Gesundheit - ein Vergleich mit der Schwangerschaft von Jugendlichen (!): Natürlich lässt sich eine solche Schwangerschaft glücklich leben, dennoch ist es vorzuziehen, nicht zu früh schwanger zu werden.

5 Die WHO hat ihren ersten internationalen Bericht zur Behinderung herausgebracht (WHO, Genf 2011). Aus diesem geht hervor, dass gegenwärtig etwa $15 \%$ der Bevölkerung weltweit mit einer Behinderung leben. www. who.int/entity/disabilities/ world_report/2011/fr

* Jean Martin, alt Kantonsarzt (Waadt) und Mitglied der Nationalen Ethikkommission und der Redaktion.
Ein Satz, der erstaunt - er stammt von Tom Shakespeare, einem britischen Bioethiker, der für Behinderung (disability) zuständigen Abteilung der WHO, der ihn Anfang Juli in der nahe Genf angesiedelten Fondation Brocher ausgesprochen hat. Er selbst leidet unter Achondroplasie und ist daher durch seine Kleinwüchsigkeit in seinen Fähigkeiten eingeschränkt; er bewegt sich im Rollstuhl (NB: Einige der unten stehenden, von ihm vertretenen Positionen sind bemerkenswert aus dem Munde eines Behinderten und könnten - kämen sie von jemandem anderen - als Ausdruck der Banalisierung und des Mangels an Engagement gewertet werden). Er hielt einen Vortrag im Rahmen der vom Hastings Center (New York) organisierten und thematisch mit der «Verbesserung des Menschen» (enhancement) befassten Sommerakademie.

Tom Shakespeare benutzte die im Titel stehende Formulierung, als er von der Förderung der Rechte von Behinderten durch die WHO und andere sprach, dem Recht auf ein Leben, das so weit wie möglich von Zwängen befreit ist. Mir schien besagte Formulierung sehr gut zu illustrieren, was öffentliche Gesundheit (public health) ist - in der Tat jener Teil der Medizin und der Gesundheitspolitik, der das optimale Wohlergehen der Menschen in einer Gesellschaft zum Ziel hat, der so weit wie möglich für ein autonomes, «praktikables» und zufriedenstellendes Leben im eigenen Umfeld sorgen will; ein Leben, in dem der Zugriff auf Realisierungs- und Lustpotentiale, über den die meisten Artgenossen verfügen, nicht verwehrt bleibt. Shakespeare schrieb: «Behinderung ist das Produkt der Interaktion von «begrenzten` (impaired) Körpern und sozialen Umfeldern, die ausschliessen.» [1]

Barrieren stehen hier für alles, was uns blockiert, was Lebensqualität mindert. Generell denken wir in der öffentlichen Gesundheit dabei an den Zugang zu Wasser und gesunden Nahrungsmitteln, den Kampf gegen ansteckende Krankheiten, das Vermeiden von Verschmutzung jeglicher Art, die Befreiung von Abhängigkeiten von Produkten wie Tabak und anderen, wir denken an das, was unsere physische Bewegung einschränkt usw.

Natürlich kennen wir die grossen Fortschritte, die durch die Beseitigung architektonischer Hindernisse in der Öffentlichkeit, in öffentlichen oder privaten Gebäuden, erzielt wurden, durch (mechanische, elektrische oder elektronische) Hilfsmittel, dank derer man sich von einem Ort zum anderen bewegen und viele Aufgaben des Alltags bewältigen, Computer und Geräte benutzen kann. Dazu zählen auch «Prothesen», die unzureichende oder fehlende Funktionen ersetzen, vor allem im Bereich der Sensorik. Tom Shakespeare sagte zum Thema der chirurgischen Verlängerung der unteren Gliedmassen, die Kleinwüchsigen gelegentlich empfohlen wird: «Um das Licht anzuzünden, muss ich mir nicht unbedingt die Beine verlängern lassen, es genügt, wenn der Schalter tiefer gesetzt wird.» Beim Thema Gehörlosigkeit und der Mittel, mit denen man Gehörlosen helfen kann, bezieht er sich beispielsweise auf die benachteiligten Länder, die vor allem im Fokus der WHO liegen, und sagt: «Für die Zeichensprache braucht man keine Batterien.» Dabei geht es nicht darum, moderne Mittel abzulehnen.

Da es hier um öffentliche Gesundheit und im weiteren Sinne um Sozialpolitik geht, besteht das Ziel vielmehr darin, Behinderten so viele Entfaltungsmöglichkeiten wie möglich zu geben. Dazu noch eine Anmerkung: In dem Masse, in dem Gesunden neue (vor allem technische) Möglichkeiten geboten werden, sind die öffentlichen Entscheidungsträger - und die privaten Initiativen - aufgefordert, unverzüglich entsprechende Mittel in die Wege zu leiten, um auch Behinderten Zugang dazu zu gewähren. Ist dies nicht der Fall, werden für sie neue Zwänge geschaffen.

Shakespeare hat seine Zuhörer zum Lächeln gebracht, als er von Glück sprach - diesem so schwer zu fassenden Begriff. Er sprach von radikalen Philosophen, die ihre Hoffnung in ein «enhancement» setzen, in die Verbesserung des Menschen, um ihn zu einem Übermenschen (Transhumanismus) bzw. unsterblich zu machen $[2,3]$. Wir denken dabei an Namen wie Julian Savulescu, John Harris, Ray Kurzweil. Diese Personen können besessene Perfektionisten sein, Doktrinäre, die ihr Unglück in den Unzulänglichkeiten des Menschen finden - daher fragte er sich, ob er «trotz seiner Behinderung nicht glücklicher sei». Es ist zumindest richtig, dass Glück nicht primär eine physische oder eine Frage der objektiven Funktionalität ist, sondern eine Geisteshaltung, die vor allem mit der Fähigkeit zur Anpassung einhergeht. Man könnte auch sagen, «one could see the notion of disabled as differently able» (fähig, aber anders).

Etwas rätselhaft klingt der Satz: «Ein gutes Funktionieren braucht Grenzen.» Dies lässt mich an die gute Formulierung denken: «Freiheit gilt nur durch die Grenzen, die man ihr zu setzen akzeptiert.» Etwas philosophischer schlussfolgerte Shakespeare, dass Behinderte ohne Zweifel ein ausgefülltes Leben (good life) führen können, wenngleich es jedoch einfacher sei, nicht behindert zu sein $[4,5]$.

Jean Martin 